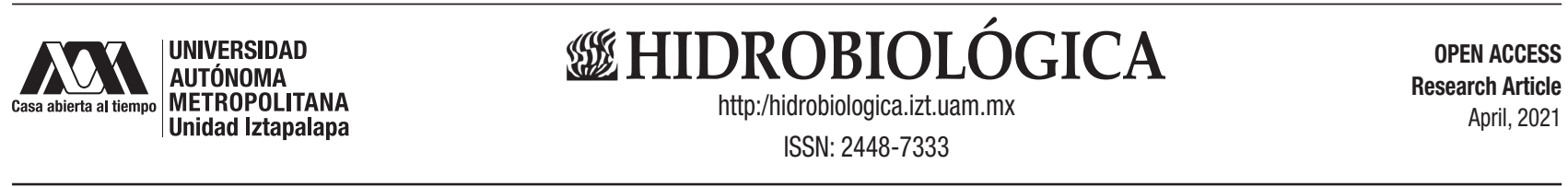

\title{
Macroinvertebrados acuáticos como bioindicadores de calidad de agua en el río Chicú, Cundinamarca, Colombia
}

\author{
Aquatic macroinvertebrates as water quality bioindicators in Chicú river, Cundinamarca, Colombia \\ María Teresa Rincón-Bell $0^{1 \oplus}$, Fabián Yesid Soler-Romero ${ }^{1 \oplus}$, Dayam Soret Calderón-Rivera ${ }^{2 \oplus}$, Ronal Jackson Sierra-Parada ${ }^{3 \oplus}$ \\ y Ángela María Jaramillo-Londoño ${ }^{2 \odot}$
}

Auxiliar de investigación-Semillero de investigación Recurso Hídrico y Territorio, Facultad de Ingeniería Ambiental, Universidad Santo Tomás. Cra. 9 \#51-11, 5878797 Bogotá, Colombia

Docente-Semillero de investigación Recurso Hídrico y Territorio, Facultad de Ingeniería Ambiental, Universidad Santo Tomás Cra. 9 \#51-11, 58787 97, Bogotá, Colombia

${ }^{3}$ Docente-Semillero de investigación iLand Facultad de Ingeniería Ambiental, Universidad Santo Tomás. Cra. 9 \#51-11, 58787 97, Bogotá, Colombia

*Corresponding author: Fabián Yesid Soler-Romero: e-mail: fabiansoler@usantotomas.edu.co

To quote as:

Rincón-Bello, M. T., F. Y. Soler-Romero D. S. Calderón-Rivera, R. J. Sierra-Parada \& Á. M. Jaramillo-Londoño. 2021 Macroinvertebrados acuáticos como bioindicadores de calidad de agua en el río Chicú, Cundinamarca, Colombia Hidrobiológica 31 (1): 17-29.

DOl:10.24275/uam/izt/dcbs/hidro/2021v31n1/ Rincon

\section{RESUMEN}

Antecedentes: Estudios realizados en la última década determinaron una evidente perturbación ambiental del río Chicú a causa del incremento demográfico en la zona y a actividades como ganadería y agricultura que ejercen presión antrópica sobre el cuerpo hídrico mediante las descargas de sus residuos líquidos al mismo. Objetivos: Determinar la calidad del agua del río Chicú con ayuda de índices bióticos (BMWP, ASPT y ABI) con base en macroinvertebrados acuáticos y analizar su relación con parámetros físicoquímicos monitoreados en los mismos sitios de muestreo biológico. Métodos: Los muestreos fueron realizados en cuatro sitios distribuidos a lo largo de la cuenca y en tres temporadas climáticas (de baja precipitación, de precipitación media y de alta precipitación). Se calcularon índices bióticos (BMWP, ASPT y ABI), índices de diversidad y se analizaron parámetros fisicoquímicos. El análisis de resultados se llevó a cabo mediante un análisis de correspondencia canónica con el fin de determinar la correlación existente entre las diferentes variables e índices. Resultados: Se identificaron 9 órdenes, 15 familias y 7093 individuos en los cuatro sitios, encontrando que la mayor cantidad de individuos pertenecen a las familias Sphaeriidae (49,71\%) y Physidae $(21,83 \%)$. A partir de los índices y las variables analizadas se determinó que la calidad del agua del río está enmarcada en un rango que va de "moderadamente contaminado" a "fuertemente contaminado". Mediante el análisis de correspondencia canónica se observó una agrupación particular de ciertas familias identificadas con los parámetros oxígeno disuelto y pH. Conclusiones: La calidad del agua en el río Chicú presenta un comportamiento decreciente desde la parte alta de la cuenca (primer sitio de muestreo) a la parte baja (cuarto sitio de muestreo), las variables oxígeno disuelto y $\mathrm{pH}$ son las que denotan una mayor sensibilidad en cuanto a la presencia de ciertas familias de macroinvertebrados acuáticos en el río.

Palabras clave: estado ecológico, índices bióticos, correspondencia canónica, macroinvertebrados acuáticos, calidad del agua, río Chicú

\section{ABSTRACT}

Background: Studies carried out in the last decade identified an evident environmental disturbance of the Chicú river on the basis of demographic increase in the zone and activities as agriculture and livestock rearing that exert anthropic pressure over the water source through waste water discharges. Goals: Determine the water quality of the Chicú river with the help of biotic indexes (BMWP, ASPT and ABI) based on aquatic macroinvertebrates and analyze their relationship with physical and chemical parameters monitored in the same biological sampling sites. Methods: Sampling took place in four sites distributed along the river and in three intensity-based rainy seasons (low-rain season, middle-rain season, and high-rain season). Biotic indexes (BMWP-Col, ASPT and ABI), and diversity indexes were calculated, and physicochemical parameters in the water were analyzed. Canonical Correspondence Analysis were applied to determine the correlation between indexes, families and parameters. Results: Nine orders, 16 families and 7,094 individuals were identified in the sampling sites, it was found that the highest abundance of individuals belongs to the families Sphaeriidae $(49,71 \%)$ and Physidae (21,83\%). The values of the indexes BMWP and ABI showed that the water quality 
averaged from "moderately polluted" to "strongly polluted"; canonical correspondence analysis showed a specific grouping of dissolved oxygen and $\mathrm{pH}$ with some identified families. Conclusions: Water quality in the Chicú river had a decreasing trend from the high (site 1 of sampling) to the low zone (site 4 of sampling) of the basin. The parameters dissolved oxygen and $\mathrm{pH}$ are the ones that reflect a higher sensitivity to adaptability of some families of aquatic macroinvertebrates in the river.

Keywords: ecological state, biotic indexes, canonical correspondence, aquatic macroinvertebrates, water quality, Chicú river

\section{INTRODUCCIÓN}

Los ecosistemas dulceacuícolas tienen gran relevancia para el bienestar del ser humano por los servicios ecosistémicos que ofrecen, como son el abastecimiento, progreso económico, y uso recreacional y cultural; pero de forma simultánea continuamente se ven sometidos a diversas presiones antrópicas (Meza et al., 2012).

Estos ecosistemas se destacan por poseer biodiversidad rica y variada de peces y otros vertebrados, y una mayor diversidad de invertebrados, plantas y algas. En Colombia, un gran número de ríos y otros sistemas acuáticos continentales, sobre todo en la región Andina, en donde se concentra la mayor parte de la población del país, están sometidos a diversos impactos antrópicos que se relacionan en gran medida con cambios en la cobertura vegetal y uso de suelo, lo cual condiciona la calidad y volumen de agua en los cuerpos hídricos y el suministro de bienes y servicios ecosistémicos para la sociedad (Guevara, 2014).

La subcuenca del río Chicú hace parte de la cuenca alta del río Bogotá localizada en la sabana de Bogotá, en este territorio se realizan actividades económicas como el cultivo de flores, hortalizas y ganadería y a su vez esta zona comprende las áreas urbanas de los municipios de Tenjo y Tabio (Cundinamarca), estas actividades económicas afectan los ecosistemas dulceacuícolas de la cuenca. De igual manera, el río cuenta, principalmente en la zona rural, con una red de aguas superficiales denominada "vallados" que inicialmente eran usados para la escorrentía de aguas de la zona alta, pero que con el tiempo y debido a la falta de conciencia por parte de la comunidad, han sido implementados para la disposición de residuos sólidos y vertimientos de aguas residuales de fuentes domésticas y agropecuarias, lo que genera taponamientos en los cauces y por consiguiente inundaciones en épocas de altas precipitaciones. Otra problemática que afecta el río, son las malas prácticas pecuarias presentes en la mayoría de predios ganaderos (Gonzalez \& Bulla, 2018).

Así mismo, según Rodríguez \& Beltrán (2015) el municipio de Tenjo produce 44 litros por segundo (I/s) de aguas residuales, de los cuales solo $21 \mathrm{l} / \mathrm{s}$ son tratados, y $23 \mathrm{l} / \mathrm{s}$ están siendo evacuados de manera directa al caudal del río Chicú, que atraviesa el municipio. Las aguas no tratadas que finalmente llegan al río Chicú, no están cumpliendo con los objetivos de calidad de agua, contribuyendo a su vez a la contaminación y deterioro del río Bogotá.

Actualmente la bioindicación, con base en macroinvertebrados acuáticos, es considerada uno de los mejores métodos para determinar la calidad del agua, debido a que integra diferentes componentes del ecosistema que están en la capacidad de dar respuesta a las alteraciones ocasionadas por actividades humanas (Meza et al., 2012). Los integrantes de esta comunidad son sensibles a la contaminación orgánica y la degradación del hábitat, por tal razón, en la evaluación ambiental del recurso hídrico es valioso su potencial como bioindicadores de calidad del agua (Merritt \& Cummins, 1996).

Debido a los problemas ambientales que presenta el río Chicú, esta investigación busca analizar la calidad del agua del río y su relación con la influencia antrópica que recibe, y que esta información facilite la toma de decisiones por parte de entidades territoriales en cuanto a la gestión, uso y conservación de este cuerpo de agua. Este proyecto tuvo una duración de un año y cuatro meses e involucró la planeación y formulación de metodologías y planes de trabajo, visitas a campo que incluyeron toma de muestras en cada uno de los sitios de estudio, trabajo analítico en laboratorio, estructura y análisis de resultados y desarrollo del presente artículo.

Área de estudio: El proyecto se realizó en la subcuenca del río Chicú, que comprende parte de los municipios de Tabio y Tenjo, zona ubicada a aproximadamente $37 \mathrm{~km}$ de la ciudad de Bogotá (Figura 1). El río nace en el municipio de Tabio, en el lugar denominado cuchilla de Paramillo, donde recibe las aguas de las quebradas Honda, Santa Librada y Cuzá, atraviesa también el municipio de Tenjo donde recibe las aguas de las quebradas Garay, Chince, Tiguase y la Chucua, finalmente, desemboca en el río Bogotá a la altura del municipio de Cota (Empresa de servicios públicos Emsertenjo , 2018).

Se establecieron 4 sitios de muestreo distribuidos de manera estratégica a lo largo del río, con el objetivo de analizar el cambio en la calidad del agua conforme se presentan alteraciones de origen antrópico en relación con el uso y cobertura de suelo y actividades que implican descargas de aguas servidas en el cuerpo hídrico. El muestreo se realizó en el año 2017 y se tomaron muestras en tres temporadas climáticas distintas: el muestreo 1 se realizó en el mes de julio (correspondiente a temporada seca con precipitación media de $50 \mathrm{~mm}$ ), el muestreo 2 en el mes de septiembre (temporada intermedia) y el último muestreo en octubre cuando las lluvias llegan a su intensidad máxima (Molano, 1997).

El sitio 1 de muestreo se localiza en la parte alta de la cuenca y recibe las descargas de aguas residuales provenientes del municipio de Tabio, y de cultivos que descargan sus residuos líquidos al cauce. Los sitios 2 y 3 tienen una separación longitudinal de $150 \mathrm{~m}$, en el sitio 2 hay vertimientos domésticos y de un aserradero, y aguas abajo, en el sitio 3 se encuentra un predio con producción agrícola y ganadera. Finalmente, el sitio 4 se ubica en la parte inferior de la cuenca y recibe descargas de aguas residuales domésticas y aquellas derivadas de la ganadería y cultivos circundantes.

\section{MATERIALES Y MÉTODOS}

Recolección de muestras e identificación: En cada sitio de muestreo se delimitó un tramo de $10 \mathrm{~m}$ de longitud sobre el costado del cauce, en el cual se hizo un barrido a contracorriente, con una red tipo D-net de 250 micras de ojo de malla y un esfuerzo de muestreo de 10 minutos en cada orilla. Los macroinvertebrados fueron recolectados a partir de la vegetación ribereña, lodos, rocas y troncos (Roldán-Pérez, 1996).

Los ejemplares capturados fueron fijados en frascos de vidrio con alcohol industrial al $70 \%$ para su preservación y posterior análisis en el laboratorio. Se realizó la medición in situ de parámetros fisicoquímicos con una sonda multiparamétrica Hannah HI 2829, en los mismos sitios de muestreo biológico. Para la identificación y cuantificación de los 


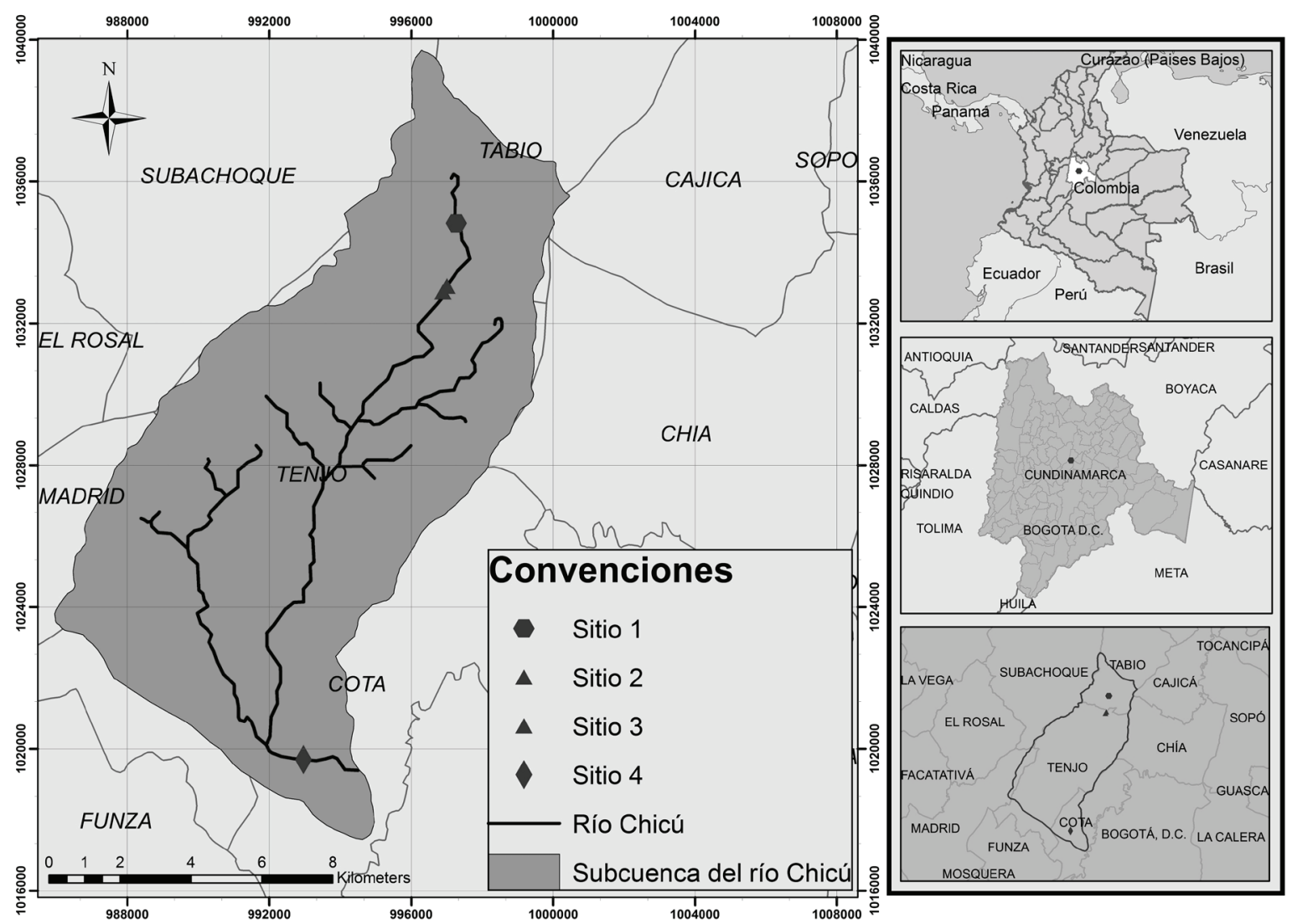

Figura 1. Mapa de localización del río Chicú (Cundinamarca) y los cuatro sitios de muestreo.

Fuente: Elaboración propia.

macroinvertebrados, se realizó una limpieza de las muestras con el fin de separar los organismos y los restos de materia orgánica. La identificación se hizo con ayuda de un estereoscopio KONUS Biorex-2 y claves taxonómicas especializadas de Roldán-Pérez (1996) y Arango-Alvarez (2005). Se observaron las características de cada individuo y se identificaron al nivel de familia, y se registró el número de individuos de cada familia por sitio y réplica de muestreo.

Análisis de datos: Los macroinvertebrados identificados se agruparon por phylum, clase, orden y familia. El conteo de individuos se hizo con la finalidad de calcular la diversidad y abundancia de cada uno de los grupos definidos. Para determinar la validez de los muestreos llevados a cabo, se realizó una curva de acumulación de familias a través del Software EstimateS versión 9.1.0, basada en el número de muestreos y la cantidad de familias encontradas. Así mismo, con la finalidad de determinar la diversidad y dominancia de los organismos identificados, se calcularon índices de dominancia de Simpson, y de equidad de Shannon-Wiener para cada sitio y temporada.

En cuanto a la bioindicación se aplicaron tres índices de calidad que involucran la presencia de familias de macroinvertebrados, entre ellos el BMWP-Col (Biological Monitoring Working Party) modificado para Colombia por Roldán-Pérez (1996), que es un método simple y rápido para evaluar la calidad del agua usando los macroinvertebrados. El método sólo requiere llegar al nivel de familia y los datos son cualitativos (presencia 0 ausencia). El puntaje va de 1 a 10 de acuerdo con la tolerancia de los diferentes individuos a la contaminación orgánica. Las familias más sensibles reciben un puntaje de 10; en cambio, las más tolerantes a la contaminación reciben una puntuación de 1 (Armitage, 1992). La suma de los puntajes de todas las familias proporciona el valor total del índice BMWP-Col (Roldán, 2016). Los demás índices de análisis fueron ASPT (Average Score per Taxon), que se calcula dividiendo la puntuación total del BMWP-Col en el número de los taxones calificados en la muestra, lo cual expresa el promedio de indicación de calidad del agua que tienen las familias en un sitio determinado; y $\mathrm{ABI}$ (Andean Biotic Index) que es un índice basado en el BMWP y se aplica en zonas de los Andes sobre los 2000 m.s.n.m (Cordero-Ledergerber, 2015).

Los datos obtenidos en los cálculos de los índices de calidad, diversidad y dominancia y los parámetros fisicoquímicos monitoreados en campo fueron sometidos a una prueba de Kruskal-Wallis, que es un método no paramétrico que evalúa si dos o más muestras se extraen de la misma distribución y si las diferencias entre sus datos son estadísticamente significativas 0 no (Zhang \& Sun, 2017). Los mismos datos fueron usados para realizar un diagrama de cajas y bigotes por cada variable a nivel espacial y temporal, es decir cada variable fue graficada con sus valores a nivel de sitio y temporada de muestreo, con la finalidad de observar la distribución y tendencia que presentan los datos. 
Finalmente, por medio del software Rstudio versión 1.3.1073, se realizó un ACC (Análisis de Correspondencia Canónica), que es una técnica de análisis de datos que extrae la estructura de correlación multidimensional entre dos conjuntos de variables (Qinqin \& Qin, 2017). El ACC fue empleado para determinar la correlación existente entre los parámetros fisicoquímicos, los índices de calidad, las familias identificadas y los índices de Shannon y de Simpson, en cada uno de los sitios y temporadas de muestreo a partir de bases de datos estructuradas por sitios y por temporada de muestreo.

Con el fin de determinar la relación existente entre el uso y cobertura de suelo, la calidad determinada a partir de los índices, lo observado en campo y los análisis correspondientes a cada uno de los sitios de muestreo, se realizó un mapa que ilustra las condiciones del suelo de la subcuenca del Rio Chicú, a través del Software ArcGIS versión 10.5, implementando un archivo Shapefile con datos relacionados al uso y cobertura de suelo (SIGOT, 2018).

\section{RESULTADOS}

Estructura de la comunidad de macroinvertebrados acuáticos: En las tres temporadas de recolección de muestras, los sitios de muestreo se caracterizaron por una hidrodinámica lenta, con bajas velocidades y flujo poco turbulento, con abundante presencia de vegetación sumergida y lodo.

Se identificaron 16 familias pertenecientes a 9 órdenes, 5 clases y 3 phyla para un total de 7094 individuos en los cuatro sitios y en las tres temporadas de muestreo (Tabla 1).
El sitio 1 presentó la mayor abundancia de familias y el menor número de individuos en comparación a los demás. La familia ErpobdeIlidae (sanguijuelas) es la que registró mayor abundancia.

El sitio 2 presentó un mayor número de individuos que el 1, y se ve una disminución paulatina en cuanto al número de familias encontradas. Physidae (caracoles) y Sphaeriidae (almejas) fueron las familias con mayor abundancia en este sitio. En el sitio 3 se ve una disminución del número de individuos presentes, en comparación con el sitio 2, pero las familias y la cantidad de estas fue muy similar.

El sitio 4 fue el que presentó el menor número de familias, pero la cantidad de individuos fue mayor. La familia Sphaeriidae fue la que registró mayor abundancia en este sitio.

Se puede observar que la primera temporada (baja precipitación) presentó el menor número de individuos, y en la tercera temporada (alta precipitación) se observó una mayor cantidad de individuos que en las demás.

El número más alto de individuos encontrados pertenece a la familia Sphaeriidae, con un $49,71 \%$ sobre el total de la población muestreada. Physidae fue la segunda familia con mayor cantidad de individuos presentes, con un $21,83 \%$, y aunque es un porcentaje significativo en comparación con el resto de familias, aún sigue siendo aproximadamente la mitad de la abundancia que posee la familia Sphaeriidae (Figura 2). De igual manera, la Tabla 1 describe cómo estas dos familias con mayor abundancia están presentes en casi todos los sitios y temporadas de muestreo.

Tabla 1. Clasificación taxonómica de los individuos identificados (M: Temporada de muestreo; P: Sitio de muestreo).

\begin{tabular}{|c|c|c|c|c|c|c|c|c|c|c|c|c|c|c|c|}
\hline \multirow{2}{*}{ PHYLUM } & \multirow{2}{*}{ CLASES } & \multirow{2}{*}{ ORDEN } & \multirow{2}{*}{ FAMILIAS } & \multicolumn{5}{|c|}{ M1 } & \multicolumn{4}{|c|}{ M2 } & \multicolumn{3}{|c|}{ M3 } \\
\hline & & & & P1 & P2 & P3 & P4 & P1 & P2 & P3 & P4 & P1 & P2 & P3 & P4 \\
\hline \multirow{2}{*}{ Annelida } & \multirow{2}{*}{ Clitellata } & Arhynchobdellida & Erpobdllidae & 0 & 195 & 18 & 0 & 345 & 15 & 0 & 61 & 46 & 205 & 17 & 2 \\
\hline & & Rhynchobdellida & Glossiphoniidae & 5 & 0 & 0 & 0 & 4 & 0 & 0 & 4 & 4 & 29 & 3 & 0 \\
\hline \multirow{3}{*}{ Mollusca } & \multirow{2}{*}{ Gastropoda } & \multirow{2}{*}{ Basommatophora } & Physidae & 58 & 0 & 76 & 15 & 3 & 21 & 14 & 710 & 12 & 409 & 34 & 197 \\
\hline & & & Planorbidae & 36 & 0 & 19 & 44 & 0 & 17 & 4 & 40 & 0 & 101 & 23 & 32 \\
\hline & Bivalvia & Venerida & Sphaeriidae & 54 & 2 & 12 & 374 & 1 & 45 & 21 & 1020 & 0 & 500 & 62 & 1436 \\
\hline \multirow{11}{*}{ Arthropoda } & \multirow{5}{*}{ Malacostraca } & \multirow{3}{*}{ Amphipoda } & Gammaeridae & 0 & 0 & 0 & 0 & 0 & 0 & 0 & 38 & 0 & 52 & 1 & 97 \\
\hline & & & Hyalellidae & 52 & 25 & 9 & 0 & 62 & 0 & 0 & 50 & 63 & 69 & 6 & 24 \\
\hline & & & Ephydridae & 1 & 0 & 0 & 0 & 0 & 0 & 0 & 0 & 0 & 0 & 0 & 0 \\
\hline & & \multirow[t]{3}{*}{ Diptera } & Tipulidae & 0 & 0 & 0 & 0 & 0 & 0 & 0 & 0 & 1 & 0 & 0 & 0 \\
\hline & & & Chironomidae & 137 & 6 & 0 & 0 & 0 & 0 & 0 & 0 & 0 & 12 & 0 & 0 \\
\hline & & & Notonectidae & 0 & 0 & 0 & 0 & 1 & 0 & 0 & 1 & 1 & 0 & 0 & 0 \\
\hline & \multicolumn{15}{|l|}{ Insecta } \\
\hline & & \multirow[t]{2}{*}{ Hemiptera } & Corixidae & 0 & 0 & 0 & 0 & 8 & 0 & 0 & 3 & 3 & 1 & 0 & 0 \\
\hline & & & Aeshnidae & 0 & 0 & 0 & 0 & 0 & 0 & 0 & 0 & 2 & 0 & 0 & 0 \\
\hline & & Odonata & Hydrophillidae & 0 & 0 & 0 & 0 & 2 & 0 & 0 & 0 & 0 & 0 & 0 & 0 \\
\hline & & Coleoptera & Dytiscidae & 2 & 0 & 0 & 0 & 5 & 0 & 0 & 0 & 14 & 0 & 0 & 0 \\
\hline
\end{tabular}

Fuente: Elaboración propia. 


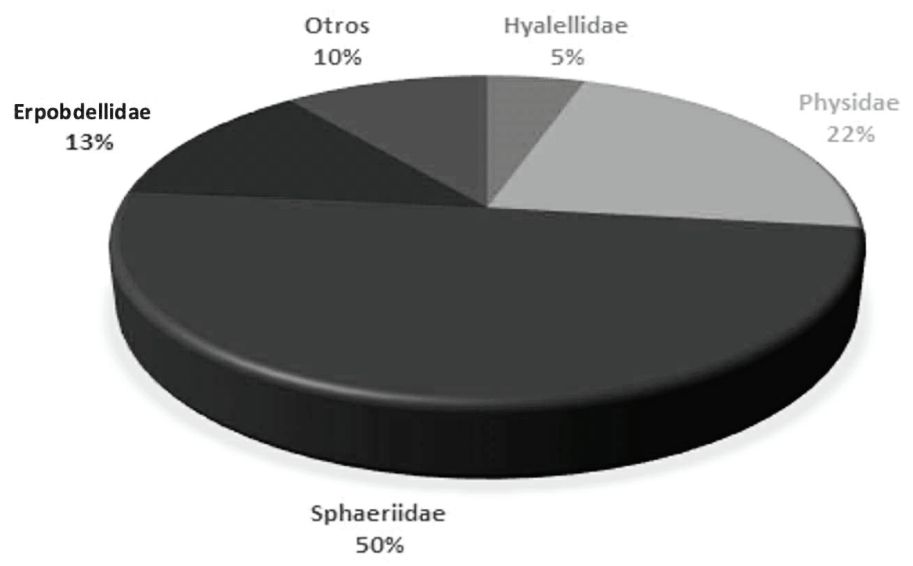

Figura 2. Abundancia de familias.

Fuente: Elaboración propia.

Curva de acumulación: A través del Software EstimateS se obtuvo la curva de acumulación de familias de macroinvertebrados, con el fin de evaluar la eficiencia del muestreo realizado, al verificar que el número de muestras fuese representativo. En el eje $X$ se muestra el esfuerzo de muestreo efectuado (12 unidades de esfuerzo), y el eje $Y$ representa el número de familias encontradas para cada nivel de muestreo dado (16). Cuanto mayor es el esfuerzo de muestreo, mayor es el número de taxones recolectados, así mismo las 16 familias muestreadas se encuentran dentro del intervalo de confianza con un límite superior de 21 familias y uno inferior de 12, lo que indica que el tamaño de muestra fue suficiente y representativo, así como también el número de muestreos realizados (Figura 3).

Índices de diversidad: Los valores obtenidos en el cálculo de los índices de diversidad de Shannon-Wiener y de Simpson se muestran en la tabla 2, los cuales oscilan entre 0.1866 y 1.8831 , un indicio de que la diversidad de familias presente en el río Chicú es baja y que se presenta alta dominancia en la mayoría de los sitios y temporadas de muestreo, ya que según Moreno (2001) para el índice de Simpson, los valores dentro del rango de 0-0.5 indican "muy baja diversidad o "muy alta dominancia" y los que se encuentran en el rango $>0.5-0.7$ caracterizan "baja diversidad" o "alta dominancia", y en la Tabla 2 se puede observar cómo los valores en las tres temporadas de muestreo y en Ios cuatro sitios oscilan entre 0.2 y 0.7 , lo que hace referencia a aguas con alta dominancia; de igual manera se establece que para el índice de Shannon, los ecosistemas con valores $\leq 1$ representan comunidades con "muy baja diversidad" y los que se encuentran entre $>1-1.8$ corresponden a comunidades con "baja diversidad", y en la Tabla 2 se pueden observar valores entre 0.5 y 1.8 , lo que determina aguas con baja diversidad en el cuerpo hídrico objeto de estudio.

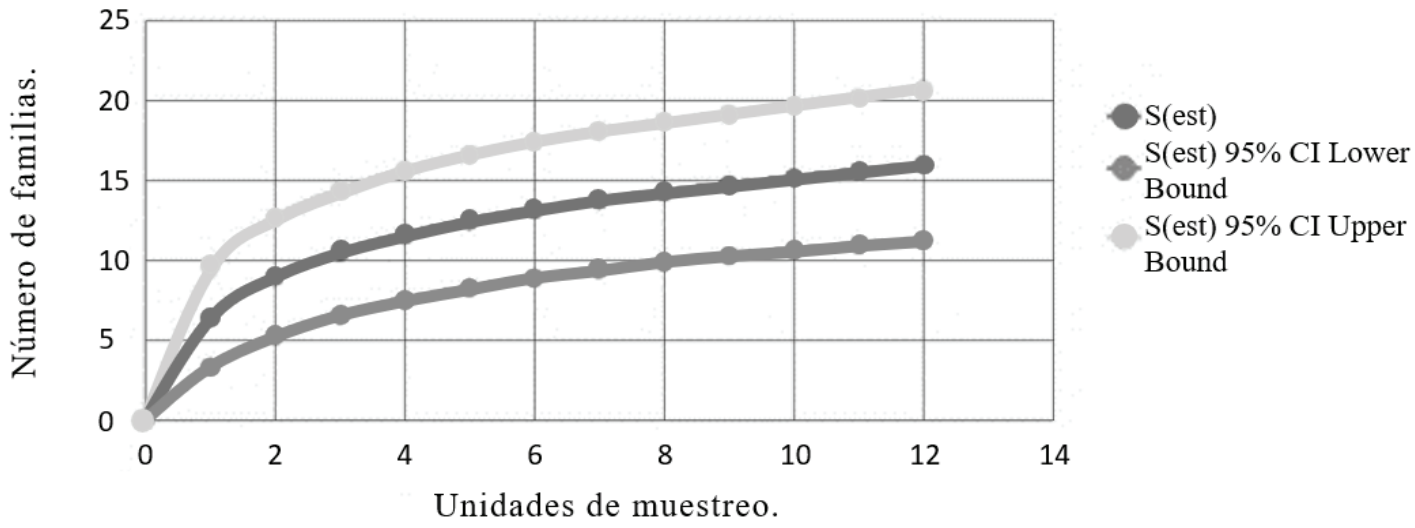

Figura 3. Curva de Acumulación de familias.

Fuente: Elaboración propia. 
Tabla 2. Valores resultantes de Índices de Diversidad.

\begin{tabular}{cccc}
\hline MUESTREO & SITIO & $\begin{array}{c}\text { Diversidad de } \\
\text { Shannon-Wiener }\end{array}$ & $\begin{array}{c}\text { Dominancia } \\
\text { de Simpson }\end{array}$ \\
\hline $\mathbf{1}$ & $\mathbf{1}$ & 1,5860 & 0,2443 \\
& $\mathbf{2}$ & 0,5134 & 0,7443 \\
& $\mathbf{3}$ & 1,2657 & 0,3724 \\
$\mathbf{2}$ & $\mathbf{4}$ & 0,4754 & 0,7576 \\
& $\mathbf{1}$ & 0,7432 & 0,6560 \\
& $\mathbf{2}$ & 1,8831 & 0,1866 \\
& $\mathbf{3}$ & 0,9347 & 0,4293 \\
& $\mathbf{4}$ & 1,0933 & 0,4184 \\
& $\mathbf{1}$ & 1,6714 & 0,2594 \\
& $\mathbf{2}$ & 1,6048 & 0,2517 \\
& $\mathbf{3}$ & 1,4897 & 0,2751 \\
& $\mathbf{4}$ & 0,7147 & 0,6606 \\
\hline
\end{tabular}

Fuente: Elaboración propia.

En particular, el sitio 1, ubicado en el municipio de Tabio, presentó la mayor diversidad pero su puntaje hace que se categorice como "alta dominancia", a pesar de que es donde se presentó menor dominancia en toda el área de estudio. El sitio 2 mantuvo esta tendencia, sin embargo, presentó una disminución en cuanto a la diversidad. Los sitios 3 y 4 son los que presentaron la mayor dominancia, clasificándose como "muy alta dominancia" y a su vez con "muy baja diversidad".

Calidad de agua: Para la determinación de la calidad de agua en los cuatro sitios y en las tres temporadas, se calcularon los índices bióticos BMWP-Col (Biological Monitoring Working Party-Colombia), ABI (Andean Biotic Index) Y ASPT (Average Score per Taxon).

Índice biótico BMWP-Col: En la figura 4 se observa la variación de la calidad del agua a lo largo de los sitios y en su respectiva temporada

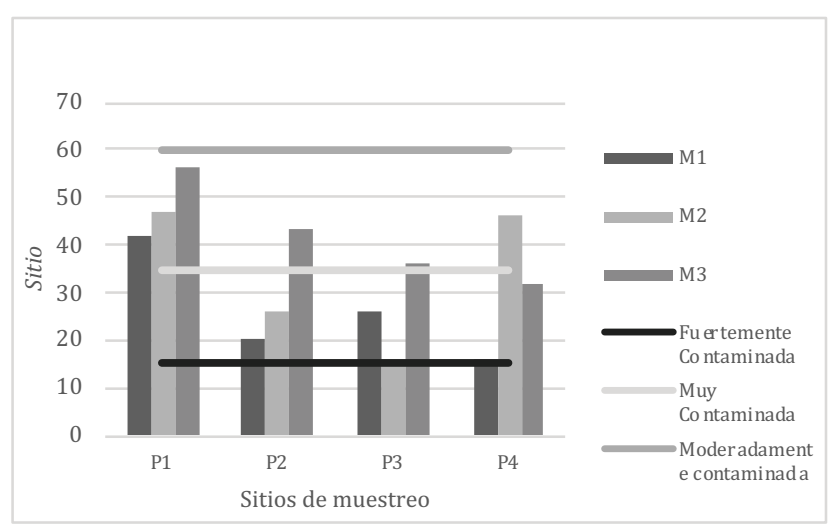

Figura 4. Valores del índice BMWP.

(Biological Monitoring Working Party for Colombia) en el periodo de muestreo.

Fuente: Elaboración propia. de muestreo. El sitio 1, en las tres temporadas de muestreo, presentó una calidad de agua con una contaminación menor que los demás, categorizado como "moderadamente contaminado". El sitio 2 mostró una disminución de calidad con respecto al 1, clasificando sus aguas como "muy contaminadas".

El sitio 3 y el sitio 4 presentaron los valores más bajos de calidad, pero en particular el sitio 4 fue el que presentó las peores condiciones de calidad, pues en la primera temporada de muestreo, sus aguas llegaron a ser "fuertemente contaminadas".

El muestreo 1 tuvo las peores características de agua en comparación con el segundo y el tercero, de igual forma se puede observar que no existe ningún sitio en ninguna temporada de muestreo donde el agua haya presentado condiciones de calidad aceptables. En general se presentaron valores que oscilan entre 16 y 56 , lo que indica que el agua del río se caracteriza por presentar mala calidad.

Índice ASPT (Average Score per Taxon): Los valores del índice ASPT indican una mejor calidad de agua con respecto a los otros índices aplicados; de acuerdo con este índice en la mayor parte de los sitios de muestreo se presentaron características de agua moderadamente contaminada.

Índice ABI (Andean Biotic Index): Los valores del índice ABI, presentaron una tendencia muy similar a la del índice BMWP-Col. La Figura 5 muestra la variación del índice $A B I$ a lo largo de los cuatro sitios en su respectiva temporada.

El sitio 1 presentó condiciones de agua categorizada como "regular", aun así, es el que presenta mejores condiciones de calidad de agua con respecto a los demás. Para el sitio 2, como también lo indicó el índice BMWP-Col, se evidenció una disminución de la calidad de agua, llegando a catalogarse como "mala". El sitio 3 presentó una calidad de agua "muy mala" en la segunda temporada de muestreo, pero en las demás su categorización fue de "mala" calidad. El sitio 4 fue el que presentó las peores condiciones de calidad, caracterizando el agua como de "mala calidad". Con este índice también se observa la misma tendencia decreciente evidenciada para el BMWP-Col, de tal manera que la primera temporada (muestreo 1) presentó las peores características de calidad del agua, y la tercera temporada (muestreo 3) las mejores.

Prueba de Kruskal-Wallis: Con el fin de determinar las diferencias estadísticas existentes para cada uno de los valores correspondientes a cada variable, se aplicó la prueba de Kruskal-Wallis, relacionando dichas variables tanto a nivel espacial como temporal. En general, no se observaron diferencias estadísticamente significativas para la mayoría de las variables; la única que presentó tales diferencias fue el pH, para el cual se observó una diferencia estadísticamente significativa con un valor de significancia de 0.0218 (Tabla 4); esto indica que los valores de $\mathrm{pH}$ tuvieron una alta variación entre cada temporada. A pesar de que la variable saturación de oxígeno disuelto arrojó un valor de diferencia menor a 0.05 (valor umbral), se considera que, por su alta cercanía a este valor, presenta diferencias estadísticamente significativas a nivel espacial (0.082), es decir, que los valores de oxígeno disuelto son diferentes significativamente entre cada sitio.

Diagramas de cajas y Bigotes: La figura 6 muestra el comportamiento temporal y dispersión de los datos de las variables físicoquímicas, índices bióticos e índices de diversidad y dominancia. Se puede ob- 
Tabla 3. Valores del Índice ASTP (Average Score per Taxon) en el periodo de muestreo.

\begin{tabular}{ccccc}
\hline & \multicolumn{4}{c}{ INDICE ASTP } \\
\hline M1 & P1 & P2 & P3 & P4 \\
M2 & 5,3 & 5 & 5,2 & 5,33 \\
M3 & 5,1 & 5,2 & 5,3 & 5,1 \\
\hline
\end{tabular}

Fuente: Elaboración propia.

servar la tendencia de los datos y su rango de dispersión en las tres temporadas de muestreo con relación a cada una de las variables analizadas. En particular a nivel temporal se observa que las variables tienen relativamente alta dispersión, pero la tendencia no es clara y varía ampliamente.

Los paneles A y B (índice de Shannon y de Simpson, respectivamente) presentan un comportamiento contrario debido a que son índices que representan características opuestas (riqueza y dominancia), Ios paneles $\mathrm{C}$ y $\mathrm{E}$, índices bióticos BMWP y $\mathrm{ABI}$, denotan un comportamiento de aumento de la calidad del agua de la primera a la tercera temporada de muestreo. Los parámetros $\mathrm{pH}$ y oxígeno disuelto ilustrados en los paneles $\mathrm{F} \mathrm{y} \mathrm{H}$, respectivamente, presentan un alza en sus valores, del primero al último muestreo, lo cual concuerda con la tendencia de calidad observada en los índices bióticos BMWP y ABI. Para la variable de $\mathrm{pH}$, en particular, se puede observar que presenta baja dispersión en sus datos.

Tabla 4. Valores de significancia para cada variable.

Obtenidos a través de la prueba de Kruskal - Wallis.

\begin{tabular}{ccc}
\hline & \multicolumn{2}{c}{ Valores } \\
\hline VARIABLE & Sitios & Muestreancia \\
SHANNON & 0.3395 & 0.4374 \\
SIMPSON & 0.3395 & 0.6677 \\
BMWP & 0.1824 & 0.2578 \\
ASTP & 0.4415 & 0.3699 \\
ABI & 0.3809 & 0.1571 \\
pH & 0.8865 & 0.0218 \\
T & 0.5857 & 0.4699 \\
OD & 0.354 & 0.122 \\
OD_SATURACIÓN & 0.08238 & 0.2189 \\
TDS & 0.2815 & 0.5004 \\
TURBIEDAD & 0.08199 & 0.4299 \\
CONDUCTIVIDAD & 0.2815 & 0.5004 \\
ORP & 0.5566 & 0.7939 \\
SALINIDAD & 0.2425 & 0.8285 \\
\hline
\end{tabular}

Fuente: Elaboración propia.

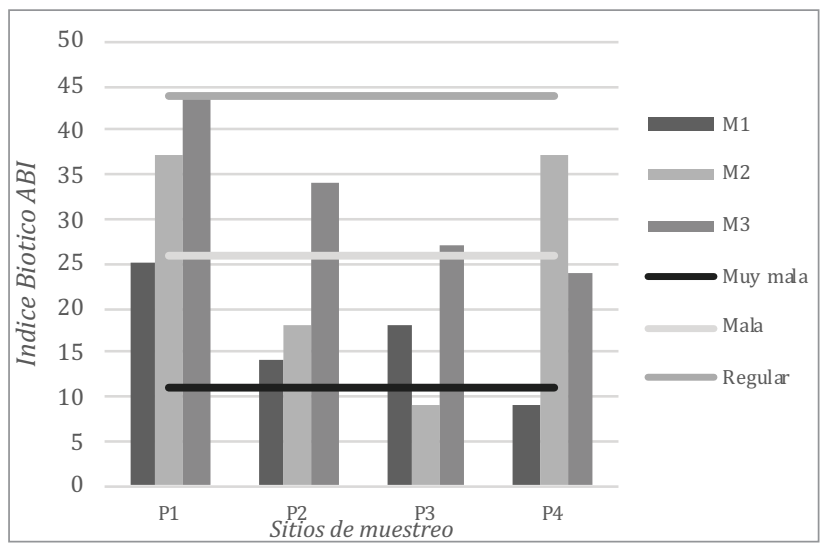

Figura 5. Variación del Índice ABI (Andean Biotic Index).

Fuente: Elaboración propia.

La figura 7 presenta los paneles que indican el comportamiento espacial de las diferentes variables objeto de estudio: índices y parámetros físicoquímicos, relacionándolos con los cuatro sitios de muestreo presentes en cada uno de los diagramas. En estos esquemas se observa que existe menor dispersión de los datos y una variación de estos más clara. En los paneles C y D se observa una tendencia de disminución de calidad del primero al último sitio de muestreo, los índices de diversidad y dominancia, como es de esperar, describen un comportamiento contrario, es decir, el índice de Shannon (diversidad) presenta una tendencia decreciente del primero al último sitio de muestreo y el índice de Simpson (dominancia) un comportamiento creciente del primero al último sitio. De acuerdo con los paneles $\mathrm{H}, \mathrm{J}, \mathrm{L}, \mathrm{N}$ se observa una tendencia contraria entre los valores monitoreados de oxígeno disuelto y las variables TDS (sólidos disueltos), conductividad y salinidad enmarcados en una variación espacial.

Análisis de correspondencia canónica: Para determinar la relación existente entre las familias identificadas y los parámetros físicoquímicos monitoreados, se realizó un análisis de correspondencia canónica (Figura 8), con ayuda del software RStudio en el que fueron incluidos los índices de calidad, diversidad, y dominancia calculados. A nivel general, las variables incluidas en la gráfica obtuvieron alta variación, ya que entre más alejadas del eje central se encuentren, mayor variación presentan y por ende se puede describir su comportamiento.

Se observa en el cuadrante superior derecho, que el muestreo dos en el primer sitio, presenta correlación con las variables oxígeno disuelto (OD) y turbiedad, de igual manera se ve la distribución de las familias Hyalellidae, Erpobdellidae y Corixidae, las cuales son afines y subsisten en dichas condiciones. En este mismo cuadrante se puede observar la presencia del índice BMWP, lo que hace referencia a una relación con el OD y las familias presentes. De igual forma, se puede observar en el cuadrante inferior derecho, valores opuestos a los parámetros sólidos totales disueltos, salinidad y conductividad presentes en el muestreo 3 y sitio 1, lo cual indica condiciones de calidad afines a las familias Planorbidae, Physidae y Glosiponidae, las que se encuentran presentes en este mismo cuadrante. En el cuadrante superior izquierdo se evidencian características opuestas a las variables 0D, pH y temperatura, lo que se relaciona con la presencia de las familias Sphaeriidae, Gammaeridae y Chironomidae. Se puede observar que la ubicación de los índices Shannon y Simpson es inversa, así como el índice ASPT y ABI. 
A nivel espaciotemporal, se observa que en el muestreo 2, la temporada de precipitación media, se caracteriza por presentar los valores monitoreados más altos de conductividad y salinidad. De igual manera, la temporada de lluvias bajas (M1) presenta los mayores valores de OD en comparación con las otras temporadas.

Cobertura de suelo: En la Figura 9 se observa que las zonas aledañas al cauce del río Chicú tienen predominancia por territorios agrícolas y en menor medida por pastizales en los que se desarrollan actividades como ganadería. El sitio 1 se encuentra bajo una influencia antrópica que determina en gran medida la calidad del agua, debido a la ganadería y floricultura allí desarrollada, actividades cuyos residuos líquidos van a parar al cuerpo de agua. Los sitios 2 y 3 se encuentran bajo la influencia ganadera y de vertimientos domésticos de recintos rurales que vierten sus aguas residuales al cauce sin un tratamiento adecuado. El último sitio de muestreo ubicado en la parte baja de la cuenca, pre- senta condiciones de cobertura de suelo similar en cuanto a pastizales y ganadería circundante y vertimientos de origen doméstico, sitio en el que se presenta una calidad del agua inferior.

\section{DISCUSIÓN}

A nivel espacial, el sitio 1 es el que tiene mejores condiciones de calidad de agua en comparación con los demás sitios de muestreo, puesto que los parámetros fisicoquímicos monitoreados allí también indican agua con menor contaminación, debido a que es en donde se presentan mayores concentraciones de oxígeno disuelto y el sitio se localiza a una mayor altitud en la cuenca, además de que no está sometido a toda la carga contaminante que recibe el río aguas abajo y presenta condiciones hidráulicas favorables. Dichas condiciones podrían incidir en el mantenimiento de variables necesarias para la supervivencia de las
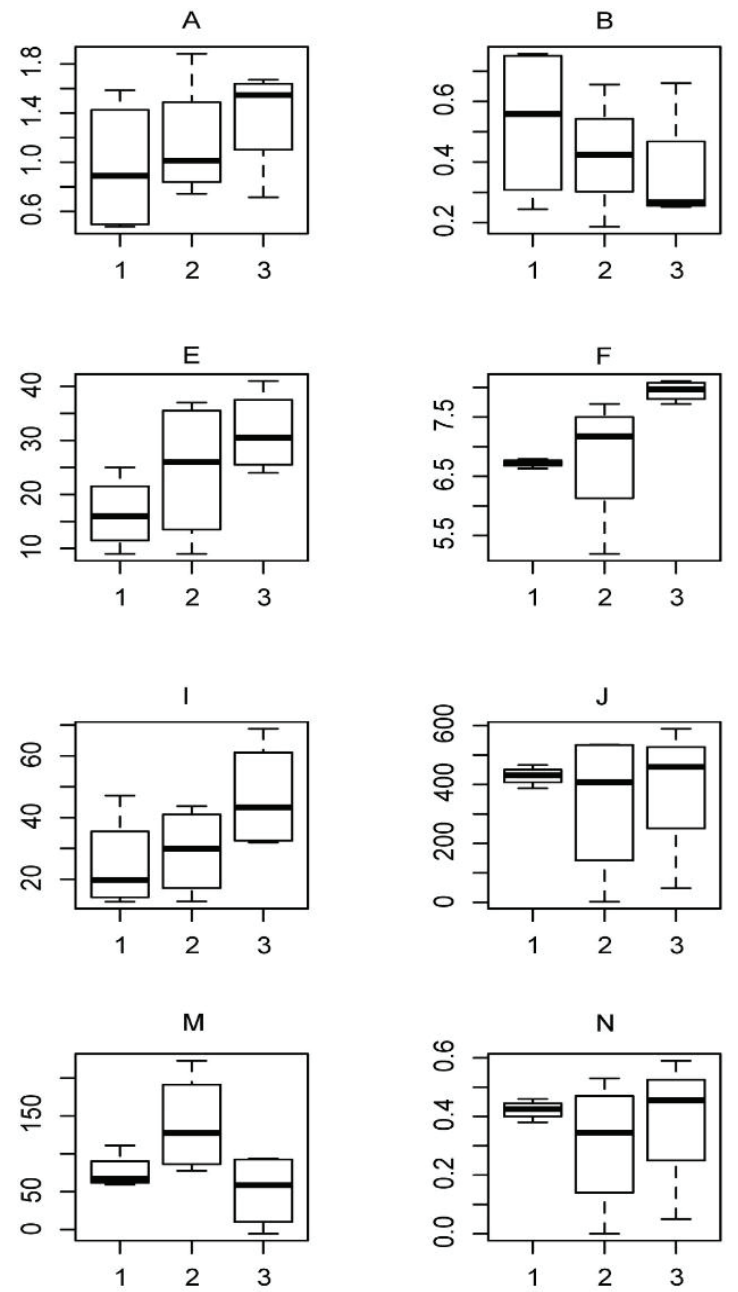
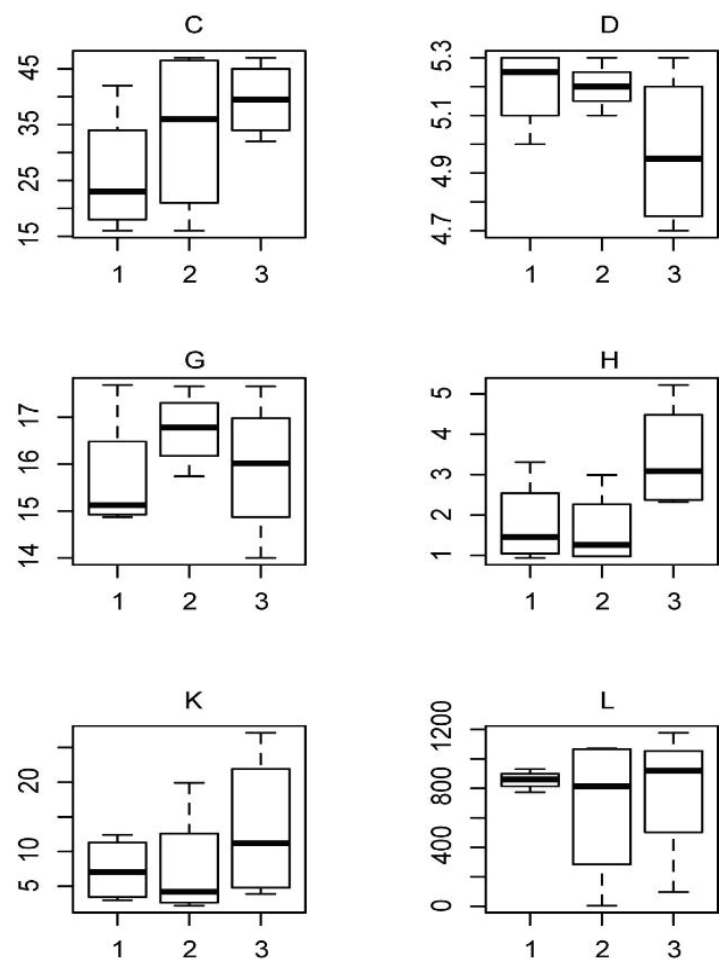

Figura 6. Variación temporal - Diagramas de cajas que relacionan temporadas de muestreo con índices, familias y parámetros. Ejes X: Temporada de muestreo Ejes Y: Rango de la Variable.

A. Índice de Shannon B. Índice de Simpson C. Índice BMWP D. Índice ASTP E. Índice ABI F. pH G. Temperatura H. Oxígeno disuelto I. turación de oxígeno J. TDS K. Turbiedad L. Conductividad M. ORP N. Salinidad.

Fuente: Elaboración propia. 

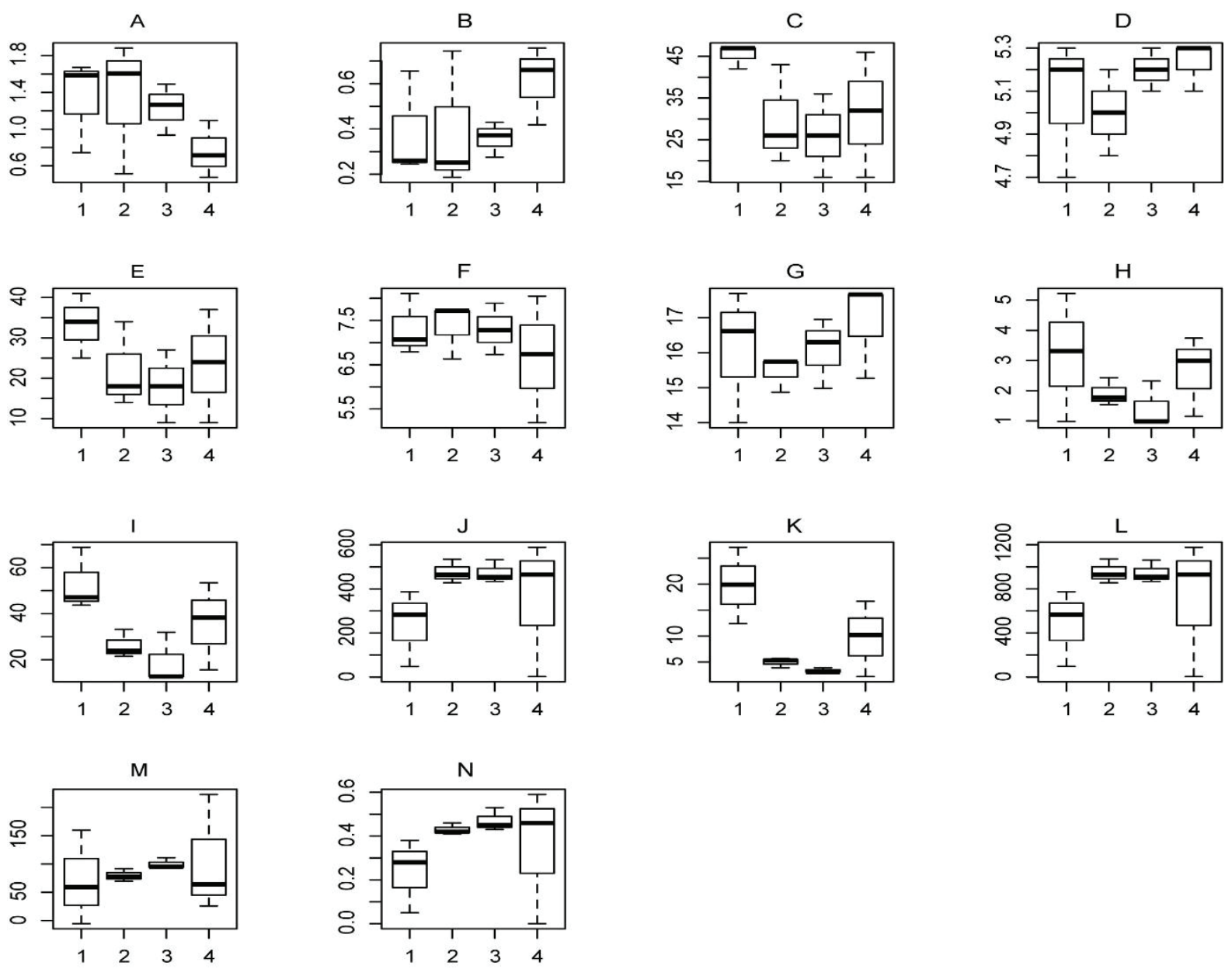

Figura 7. Variación espacial - Diagramas de cajas que relacionan sitios de muestreo con índices, familias y parámetros. Ejes X: Sitios de muestreo Ejes Y: Rango de la Variable.

A. Índice de Shannon B. Índice de Simpson C. Índice BMWP D. Índice ASTP E Índice ABI F. pH G. Temperatura H. Oxígeno disuelto I. Saturación de oxígeno J. TDS K. Turbiedad L. Conductividad M. ORP N. Salinidad.

Fuente: Elaboración propia.

familias Hyalellidae y Chironomidae, las cuales son las más abundantes en este sitio, y se caracterizan por habitar aguas someras y corrientes (Scheibler et al., 2008), así como remansos con materia orgánica en descomposición (Roldán, 2003).

El hecho de que el sitio 1 muestre una alta correlación con las variables oxígeno disuelto y saturación de oxígeno en el cuadrante superior derecho (Figura 4), no indica que este sitio se caracterice por buenas condiciones de calidad, puesto que según los índices bióticos sus condiciones aluden a aguas moderadamente contaminadas. De igual forma, en el sitio se vierten aguas residuales con contenidos de agroquímicos provenientes de cultivos de flores y hortalizas (Arroyo \& Jiménez, 2011), lo cual incide en el aumento de valores de turbiedad y el incremento de nutrientes que generan procesos de eutrofización (Bulla, 2017). En este ambiente se hacen evidentes condiciones de afinidad para la presencia de familias como Chironomidae (Diptera) y Notonectidae (Hemiptera), que son indicadoras de aguas oligomesotróficas y eutróficas (CORTOLIMA, 2009).
Algunos individuos de la familia Chironomidae se caracterizan por soportar condiciones de hipoxia (Badillo et al., 2016), lo cual se relaciona con los bajos valores de oxígeno disuelto. De igual manera, la tendencia de variación evidenciada en los diagramas de cajas y bigotes a nivel espacial, permiten observar la dinámica en la que la calidad del agua va disminuyendo desde el primero al cuarto sitio. La alta diversidad y baja dominancia que este sitio presenta también es evidenciada en la Figura 3 panel $A$, donde se muestra que el primer sitio de muestreo presenta mayores valores para el índice de Shannon.

Los sitios 2 y 3 presentan altos niveles de contaminación influenciada por actividades antrópicas que afectan las condiciones de calidad física y química del agua. Una de estas actividades, son los vertimientos domésticos, ya que el Municipio de Tenjo vierte aproximadamente el $50 \%$ de sus aguas residuales al caudal del río Chicú que atraviesa el municipio (Empresa de servicios públicos Emsertenjo , 2018). La ganadería y el constante pastoreo, son otras de las actividades más influyentes en la contaminación que se presenta en estos dos sitios, lo que 
puede incidir en los altos valores de salinidad, conductividad y sólidos suspendidos totales. Como lo indica el ACC, la conductividad presentó una alta correlación con la presencia de la familia Glosiphonidae así como lo menciona Usme \& Agudelo (2013). Por lo anterior y por las condiciones del lugar, como un fondo lodoso y bajas velocidades de flujo, se entiende la presencia y dominancia de la familia Sphaeriidae y Physidae, puesto que estas se caracterizan por soportar altos grados de contaminación orgánica, vivir en aguas estancadas y formar densas colonias (Confederación hidrográfica del Ebro, 2012). El análisis de correspondencia canónica también hace evidente la presencia de dichas familias (cuadrante inferior derecho, Figura 4) asociadas a altos valores de conductividad, salinidad y sólidos disueltos (TDS); no obstante, hay presencia de organismos de la familia Planorbidae (Basommatophora) que si bien a nivel de familia se considera que no son tolerantes a altos grados de contaminación podrían tener representantes a nivel de especie que si lo fueran (Hessberg et al., 2009).

En el sitio 4, la existencia de un mayor número de individuos y menor número de familias para las tres temporadas de muestreo guarda relación con condiciones de muy mala calidad del agua, de acuerdo con lo establecido por los índices de calidad BMWP-Col, ABI y ASTP. Los índices de Shannon y de Simpson hacen evidente que en este sitio se presenta baja diversidad y alta dominancia (Moreno, 2001), al igual que los diagramas de cajas y bigotes (Figura 3 - paneles A y B) que muestran la tendencia de disminución de diversidad a nivel espacial y los valores más altos del índice de Simpson (dominancia). En ese sitio, al igual que los sitios 1 y 2 , se presentan vertimientos domésticos de barrios aledaños del municipio de Tenjo y una ganadería más intensiva; según el ACC, se observa en ese sitio de muestreo la presencia de valores bajos, casi nulos de oxígeno disuelto y elevada temperatura del agua, variable que según Gutiérrez (2013) disminuye con la profundidad. Lo anterior, junto con la poca profundidad del cauce, incide en las malas condiciones de calidad que se presentan según los índices bióticos calculados, los cuales caracterizan el agua como "muy contaminada" y en la presencia de familias como Physidae (Hygrophila), que es la más abundante y dominante en este sitio y es representativa de aguas con importantes niveles de contaminación y altos valores de alcalinidad (Gilbert, 1978), donde las concentraciones de oxígeno son bajas (Thorp \& Rogers, 2014). Según lo anterior, a nivel espacial este sitio de muestreo es el que presenta peores condiciones de calidad del agua en todo el río.

A nivel general, la familia de almejas (Sphaeriidae) se destaca por ser la más abundante en el río, es decir es la familia dominante, pues ésta es propia de ecosistemas con concentraciones de oxígeno bajas (Thorp \& Rogers, 2014), lo cual es una característica general de todos los sitios estudiados. Según la correlación vista en el ACC y las diferencias significativas que se presentaron con la prueba de Kruskal-Wallis, las variables $\mathrm{OD}$ y $\mathrm{pH}$ fueron las que obtuvieron mayor relación con las
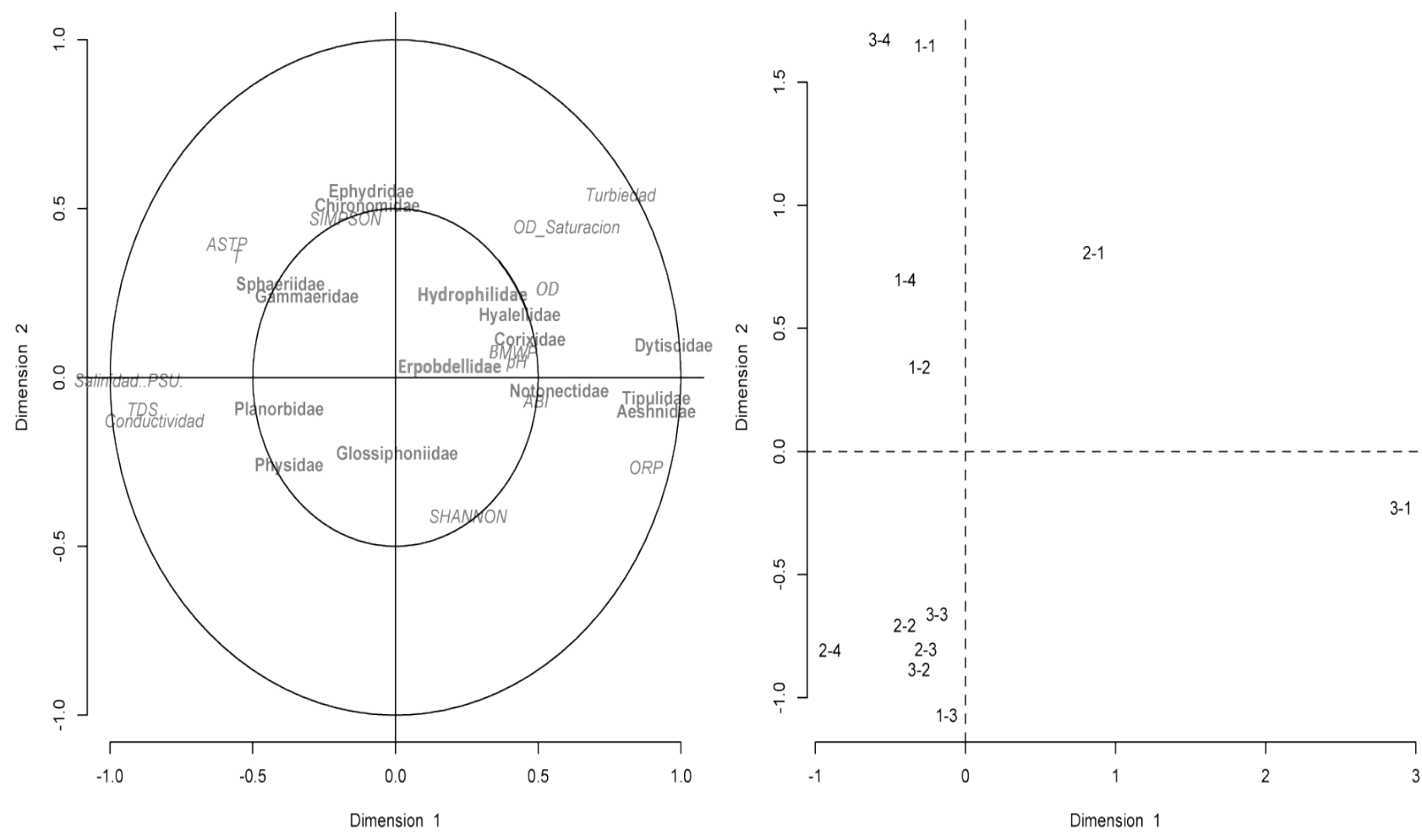

Figura 8. Representaciones en el plano definido por las variables canónicas: Variables ambientales, índices bióticos y familias (Izquierda) y temporada de muestreo - sitio de muestreo (Derecha).

Fuente: Elaboración propia. 


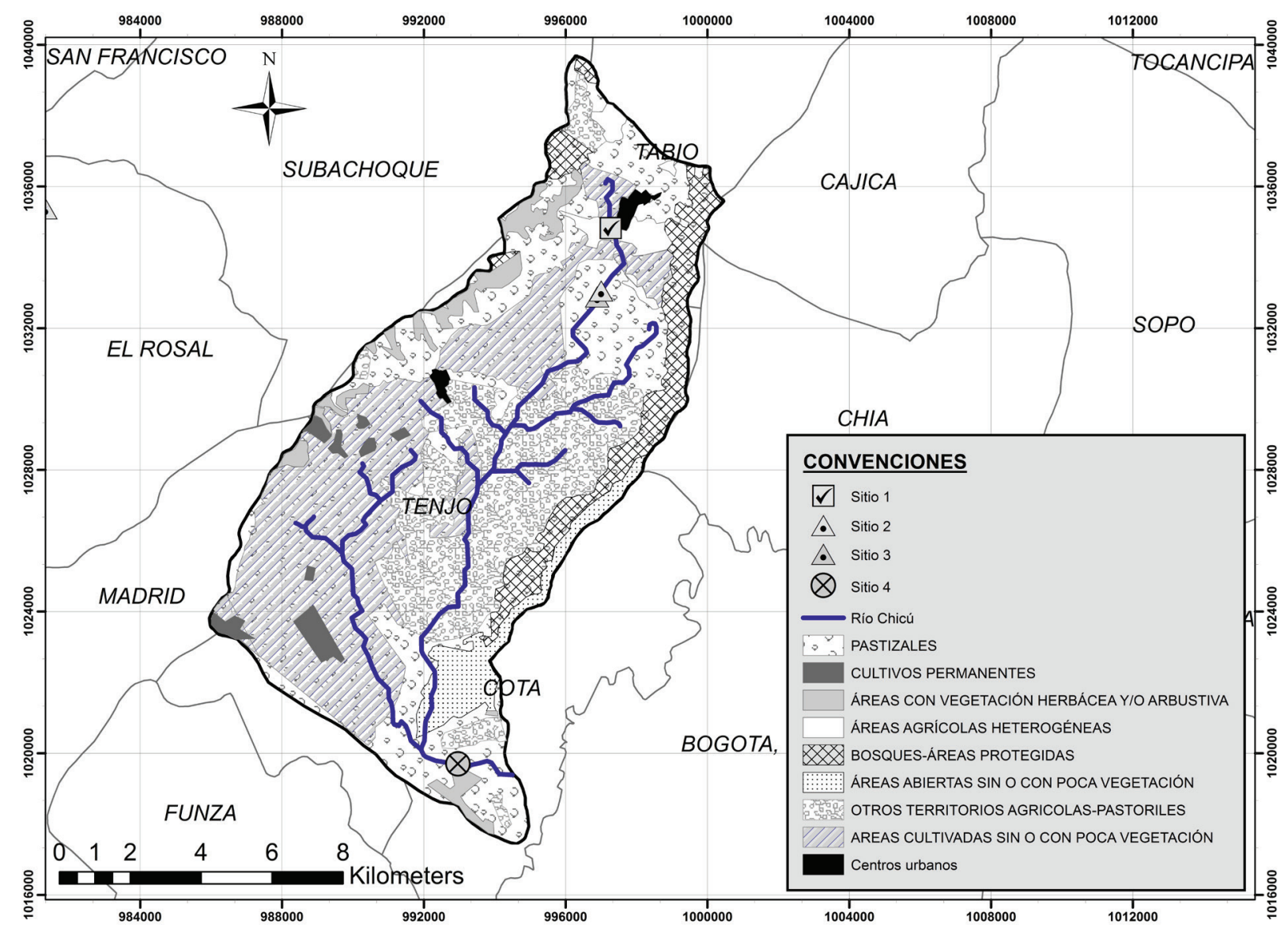

Figura 9. Mapa de cobertura de suelo en la subcuenca del río Chicú.

Fuente: Elaboración propia.

familias más abundantes del río, lo cual indica que existe afinidad entre las condiciones de calidad y la presencia de las familias que allí habitan. Así pues, se puede establecer que si los parámetros fisicoquímicos del río son alterados, la presencia 0 ausencia y el estado de las familias se verá también afectada, pues según López \& Cantillo (2013) las comunidades de macroinvertebrados sufren cambios en su composición y estructura por la acción de sustancias contaminantes, las que finalmente son las que alteran las condiciones del ambiente, es decir los parámetros fisicoquímicos.

Respecto a la valoración de la calidad ambiental analizada a través de los índices bióticos, el índice BMWP-Col, a nivel espacial en el río, se observa una tendencia de aumento de contaminación descrita por los valores obtenidos en cada sitio; la calidad del agua disminuye conforme el río va de una cota más alta a una menor, esto debido a la sumatoria de cargas contaminantes provenientes de vertimientos domésticos y agropecuarios, principalmente. Los valores arrojados por el índice $A B I$ muestran cierta tendencia de disminución de calidad del agua desde el sitio 1 hasta el 4, y su aumento a nivel temporal, donde la temporada de altas lluvias presenta las mejores condiciones de calidad. Los resultados concuerdan en gran medida con la interpretación del índice
BMWP-Col, destacando en ambos índices, la determinación del cuarto sitio de muestreo como el que presenta condiciones de calidad muy desfavorable, y la temporada de baja precipitación, como en la que se presentaron peores condiciones de calidad.

La prueba de Kruskal- Wallis para el índice BMWP-Col, ratifica estadísticamente las diferencias que existen en la calidad del agua a nivel espacial, y por ende la tendencia de disminución de esta a lo largo del río, lo que podría mostrar que los procesos de contaminación no son uniformes a nivel espacial y son capaces de alterar sensiblemente las poblaciones de algunos organismos.

A pesar de que a nivel temporal, no existen diferencias significativas en la calidad del agua, los puntajes del índice BMWP-Col muestran una tendencia de aumento de calidad del primer período de muestreo al último, ya que en el tercer período (lluvias con alta intensidad), se presentaron las mejores condiciones de calidad de agua; esto podría deberse a que la alta precipitación aumenta las concentraciones de oxígeno disuelto, lo cual influye en la dilución y autodepuración de contaminantes y por ende, mejores condiciones de calidad en el ambiente (Muñoz et al., 2015). 
De acuerdo con los análisis realizados en el desarrollo del presente artículo se puede llegar a establecer que la calidad de agua en el río Chicú no es buena y afecta en gran medida los servicios ecosistémicos provistos por este cuerpo hídrico, lo cual se ve evidenciado en factores biológicos, físicos y químicos del agua que están sometidos a la influencia del uso y cobertura del suelo que se presenta en la cuenca. Por tanto, debe ser una prioridad para entidades competentes con jurisdicción en la zona, tales como la CAR (Corporación Autónoma Regional de Cundinamarca), llevar a cabo acciones y medidas de manejo y restauración, como la reforestación en la parte alta de la cuenca con el objetivo de concentrar mayores volúmenes de agua desde esta zona, la delimitación y control sobre la ronda hídrica del río impidiendo que actividades como la ganadería intervengan en el cauce y por otro lado, llevando un control riguroso acerca de los vertimientos difusos y puntuales presentes a lo largo del río en relación al tratamiento dado a las aguas residuales de origen doméstico e industrial.

\section{AGRADECIMIENTOS}

Los autores agradecen a la Universidad Santo Tomás por la financiación del proyecto: "Evaluación de la metodología IFIM (Instream Flow Incremental Methodology) para el estudio de caudales ambientales en tres ríos de alta montaña de la cuenca del rio Bogotá, como estrategia de gestión del recurso hídrico". De igual manera agradecen al Docente Rafael Gabriel Barragán por su asesoría y orientación acerca del Análisis de Correspondencia Canónica.

\section{REFERENCIAS}

Arango-Alvarez, L. F. 2005. Metodología para la utilización de los macroinvertebrados acuáticos como indicadores de la calidad del agua. Instituto de Investigación de recursos biológicos Alexander Von Humboldt, Investigación en biodiversidad y servicios ecosistémicos para la toma de decisiones, Bogotá D.C. 263 p.

ArmitaGe, P. 1992. Biotic score and prediction to assess the effects of water abstractions on river macroinvertebrates for conservation purposes. Aquatic Conservation 2(1): 1-17. D0I:10.1002/ aqc.3270020102

Arroyo, M. M. \& D. A JimÉnEz. 2011. Evaluación de los impactos ambientales asociados a la contaminación en agua, suelo y sedimento por cromo y zinc, en los municipios de Tabio y Tenjo- Cundinamarca (Sub- cuenca del río Chicú). Tesis, Facultad de ingenierias, Programa de ingenieria ambiental y sanitaria, Universidad de La Salle. Bogota D.C. 149 p. También disponible en la página web: https://ciencia.lasalle.edu.co/ing_ambiental_sanitaria/148/?utm_ source=ciencia. Iasalle.edu.co\%2Fing_ambiental_sanitaria\%2F148\&utm_medium=PDF\&utm_campaign=PDFCoverPages

Badillo, L. R., P. R. Guayasamín, M. E. Chico \& G. J. Ortiz. 2016. Caracterización de la calidad de agua mediante macroinvertebrados bentónicos en el río Puyo en la Amazonía Ecuatoriana. Hidrobiológica 26(3): 497-507. Retrieved 5 13, 2018 from http://www.scielo.org.mx/ scielo.php?script=sci_arttext\&pid=S0188-88972016000300497

BuLLA, J. D. 2017. Formulación del plan de recuperación socio - ambiental del río Chicú en el municipio de Tenjo Cundinamarca. Facultad de Ingeniería, especialización en planeación ambiental y manejo integral de los recursos naturales, Colombia D.C. 18 p.

Confederación hidrográfica del EBro. 2012. Guía de campo Moluscos Acuáticos de la cuenca del Ebro. Madrid: Ministerio de agricultura, alimentación y medio ambiente. Gobierno de España. Disponible en línea en: http://www.chebro.es/contenido.visualizar.do?idContenido=28863 (consultado el 13 febrero 2018).

Cordero-Ledergerber, P. 2015. Calidad del agua para los ríos alto andinos, mediante indicadores biológicos. Tesis, Facultad de Ciencias exactas y naturales, Pontificia Universidad Católica de Ecuador. Quito, Ecuador. 104 p.

CORTOLIMA. 2009. Apéndice Macroinvertebrados. Ibague, Tolima. Disponible en línea en: https://www.cortolima.gov.co/sites/default/ files/images/stories/centro_documentos/pom_totare/diagnostico/ APENDICES/K_apendices2101biodiversidad/21011apendice_ macroinvertebrados/k_210apendice_macroinvertebrados.pdf (consultado el 03 mayo 2018).

Empresa de SERVIicios públicos EmSERTENJo. 2018. Empresa de servicios públicos Emsertenjo SA ESP. (E. d. Tenjo, Producer). Disponible en línea en: http://www.empsertenjo.gov.co/ (consultado el 5 noviembre 2018).

GILBERT, J. 1978. Fresh-water invertebrates of the United States. John Wiley \& Sons, New York. 803 p.

Gonzalez-Bulla, J. 2018. Formulación del plan de recuperación socio ambiental del río Chicú en el municipio de Tenjo, Cundinamarca. Tesis Universidad Milital Nueva Granada, Facultad de ingeniería, Especialización en planeación Ambiental y manejo integral de los recursos naturales, Bogotá D.C. Disponible en línea en: https:// repository.unimilitar.edu.co/bitstream/10654/16432/1/GONZALEZBULLAJUANITA2017.pdf (consultado el 05 octubre 2018).

Guevara, G. 2014. Evaluación ambiental estratégica para cuencas prioritarias de los andes colombianos: Dilemas, desafíos y necesidades. Acta Biológica Colombiana 19(1):11-24. También disponible en la página web: http://www.redalyc.org/articulo. oa?id=319029827002

Gutierréz, H. M. 2013. Ecología y medio ambiente. Guía, Univalle, Cali. Disponible en línea en: https://campusvirtual.univalle.edu.co/.../ CLASE_6_FACTORES_ABIOTICOS.pdf (consultado el 4 diciembre 2018).

Hessberg, C. M., D. R. Toro, A. G. Quintero, G. M. Quintero \& L. S. Uribe. 2009. Determinación de la calidad del agua mediante indicadores biológicas y físicoquímicos, en la estación piscícola, Universidad de Caldas, Municipio de Palestina, Colombia. Boletín Científico, centro de museos, Museo de historia natural 13(2): 89-105. También disponible en la página web: http://www.scielo.org.co/pdf/bccm/ v13n2/v13n2a06.pdf

López, J. C. \& P. L. Catillo. 2013. Biological Monitoring working party, un índice biótico con potencialidades para evaluar la calidad de las aguas en ríos cubanos. Ciencia en su PC 2: 15-25. También disponible en la página web: http://www.redalyc.org/ pdf/1813/181328708003.pdf 
MerRITT, R. \& K. Cummins. 1996. An introduction to the aquatic insects of North America. Kendall/ Hunt Publishing Company, Dubuque. Disponible en línea en: http://coweeta.uga.edu/publications/221.pdf (consultado el 05 octubre 2018).

Meza, A. M., J. Rubio, L. Diaz \& J. Walteros. 2012. Calidad de agua y composición de macroinvertebrados acuáticos en la subcuenca alta del río Chinchiná. Caldasia 34(2): 443-456.

Molano, J. 1997. Calendario Climatológico Aeronautico Colombiano. Universidad de Bogotá Jorge Tadeo Lozano, Sociedad Geográfica de Colombia. Bogotá D.C, Academia de Ciencias Geográficas. Disponible en línea en: https://www.sogeocol.edu.co/documentos/096_calen_climat.pdf (consultado el 05 noviembre 2018).

Moreno, C. 2001. Métodos para medir la biodiversidad. M \& T-Manuales y Tesis SEA, Zaragoza. 84 p.

Muñoz, H., S. Orozco, A. Vera, J. Suaréz, E. García, M. Neria \& J. Jimenéz. 2015. Relación entre oxígeno disuelto, precipitación pluvial y temperatura: río Zahuapan, Tlaxcala, México. Tecnología y ciencias del agua 6(5): 59-74.

QINoIn, Z. \& J. QIN. 2017. Concurrent quality and process monitoring with canonical correlation analysis. Journal of process control 60: 95103. D0l:10.1016/j.jprocont.2017.06.017

Rodríguez, E. C. \& J. P. Beltrán. 2015. La problemática ambiental y territorial del municipio de Tenjo (Cundinamarca): último municipio verde de la Sabana de Bogotá. Tecnogestión una mirada al ambiente 12(1): 15-26. También disponible en la página web: https://revistas. udistrital.edu.co/ojs/index.php/tecges/article/view/10562

Roldán-Pérez, G. 1996. Guía para el estudio de los macroinvertebrados acuáticos del Departamento de Antioquia. Fondo Colombiano de In- vestigaciones científicas y proyectos especiales "Francisco Jose de Caldas", Bogota D.C, Colombia. 217 p.

RoLdÁn, G. 2003. Bioindicación de la Calidad del Agua en Colombia. Uso del Método BMWP/Col. Universidad de Antioquia, Medellín. 170 p.

RoLDÁn, G. 2016. Los macroinvertebrados como bioindicadores de la calidad del agua: cuatro décadas de desarrollo en Colombia y Latinoamerica. Academia colombiana de ciencias exactas, físicas y naturales 40(155): 254-274. D0I:10.18257/raccefyn.335

Scheibler, E., V. Pozo \& A. Paggi. 2008. Dsitribución espacio-temporal de larvas de Chironomidae (Diptera) en un arroyo (Uspallata, Mendoza, Argentina). Sociedad Entomológica Argentina 67(3-4): 45-58. También disponible en la página web: http://www.scielo.org.ar/pdf/ rsea/v67n3-4/v67n3-4a05.pdf

SIGOT. 2018. Sistema de información geográfica para la planeación y el ordenamiento territorial. Disponible en línea en: http://sigotn.igac. gov.co/sigotn/frames_pagina.aspx (consultado el 3 mayo 2018).

Thorp, J. \& C. Rogers. 2014. Ecology and general Biology. Freshwater Biology and Ecology, United States of America. 25 p.

Usme, J. J. \& G. A. Agudelo. 2013. Ensamblaje de macroinvertebrados acuáticos y su relación con las variables físicas y químicas en el humedal de Jaboque-Colombia. Caldasia 35(2): 389-408. También disponible en la página web: https://revistas.unal.edu.co/index. php/cal/article/view/41209/46563

Zhang, D. \& X. Sun. 2017. Detection of image seam carving by using weber local descriptor and local binary patterns. Journal of information security and applications 36: 135-144. D0I:10.1016/j. jisa.2017.09.003 